\title{
Panorama epidemiológico de exposición a plaguicidas inhibidores de colinesterasa en 17 departamentos del país
}

\author{
Marcela Varona ${ }^{1}$, Ligia Morales ${ }^{1}$, Jaime Ortiz¹, José F. Sánchez², \\ Omayda Cárdenas ${ }^{1}$, Fernando de la $\mathrm{Hoz}^{3}$
}

\begin{abstract}
Resumen
Los organofosforados y carbamatos son los responsables de la mayor parte de intoxicaciones por plaguicidas que se presentan en el país; son potentes inhibidores de la enzima acetilcolinesterasa, causando graves intoxicaciones agudas de tipo laboral como también efectos a largo plazo.

Ante esta problemática, en 1982, el Instituto Nacional de Salud (INS), a través del Laboratorio de Salud Ambiental creó el Programa de Vigilancia Epidemiológica de plaguicidas organofosforados y carbamatos (VEO), empleando el método Lovibond para la determinación de la acetilcolinesterasa (ACHE) como indicador, con el fin de detectar precozmente los casos de absorción elevada de plaguicidas. A partir de 1993, se cuenta con información de 17 departamentos del país, datos que se analizaron.
\end{abstract}

La totalidad de las personas que participó en el estudio de 1993 a 1995 fueron 41.899, las cuales tenían antecedentes de exposición a plaguicidas organofosforados o carbamatos.

El $80 \%$ de los trabajadores que ingresaron al estudio pertenecían al género masculino, siendo el grupo de edad más afectado el de 18 a 40 años. Las mayores prevalencias de anormalidad para ACHE, según actividad económica, fue para la aplicación urbana y en relación a los oficios, para mezclador-tanqueador-formulador. Con relación a la seguridad social de 23.917 trabajadores, tan sólo $31,7 \%$ se encontraba afiliada a entidades de este tipo.

Los datos obtenidos permitieron establecer el panorama epidemiológico de la intoxicación por plaguicidas inhibidores de colinesterasa, determinando la población de mayor riesgo, lo que conllevará a plantear nuevos estudios de investigación en esta población.

\section{An epidemiological panorama of exposure to cholinesterase pesticides in 17 of the country's departments}

Organophosphorates and carbamates are responsible for the greater part of the pesticide poisonings which are presented in the country. They are potent inhibitors of the acetylcholinesterase enzyme, causing serious acute work-related poisoning as well as long term effects.

\footnotetext{
${ }^{1}$ Laboratorio de Salud Ambiental, Instituto Nacional de Salud.

${ }^{2}$ Servicio de Epidemiología Aplicada, Instituto Nacional de Salud.

${ }^{3}$ División Centro de Control de Enfermedades, Instituto Nacional de Salud.
}

Recibido para publicación: enero 30 de 1998 
In 1982, faced by this problem, the Instituto Nacional de Salud (INS), through its Environmental Health Laboratory (Laboratorio de Salud Ambiental) created the epidemiological vigilance for organophosphorate and carbamate pesticides (Programa de Vigilancia Epidemiológica de Plaguicidas Organofosforados y Carbamatos (VEO)), employing the Lovibond method to determine acetylcholinesterase (ACHE) as indicator, in order to detect cases of high pesticide absorption. From 1993 onwards, information from 17 of the country's departments was ready, this being the data which was analysed.

The total number of people who participated in the study during the years 1993 to 1995 was 41,899 , all of whom had antecedents of exposure to organophosphorate or carbamate pesticides.

$80 \%$ of the workers who participated in the study were male, the age-group most affected being 18 to 40 years old. The greatest prevalence of ACHE abnormality, according to economic activity, was for urban application and related to the jobs of mixer-sprayerwarehousing.

In relation to the social security of the 23,917 workers, only $31.7 \%$ were found to be affiliated to this type of entity.

The data obtained allowed an epidemiological panorama to be established concerning poisoning by cholinesterase inhibitor pesticides, thus determining the population at greatest risk, which will lead to new research studies of this population.

Los plaguicidas organofosforados (OF) y carbamatos $(\mathrm{C})$ comprenden un vasto grupo de compuestos de síntesis, de complejidad estructural variable, los cuales se usan a nivel agrícola, veterinario y domisanitario. Los OF son ésteres orgánicos del ácido fosfórico y de sus derivados, que comparten como característica farmacológica común y fundamental la acción de inhibir enzimas con actividad esterásica, más específicamente la inhibición de la colinesterasa en forma irreversible (1-4).

Estos plaguicidas pueden penetrar al organismo por inhalación, ingestión y a través de la piel intacta. Una vez absorbidos, se distribuyen en el organismo y sufren varias reacciones metabólicas de activación, detoxicación y conjugación, siendo eliminados relativamente rápido por vía renal. Debido a que inhiben la colinesterasa, son neurotóxicos y su mecanismo de acción tóxica es la unión e inhibición de enzimas esterásicas en forma estable $(2,4,5)$.

Los carbamatos forman parte de un gran grupo de plaguicidas que han sido desarrollados, producidos y usados en gran escala en los últimos 40 años. Son derivados del ácido carbámico por un parte, constituyendo los carbamatos propia- mente dichos y de los ácidos tiocarbámicos y ditiocarbámicos por otra, integrando el grupo de los tio y ditiocarbamatos. Se utilizan como insecticidas, herbicidas y fungicidas y son inhibidores reversibles de la colinesterasa $(2,4,5)$.

En nuestro país, la agricultura ocupa aproximadamente el $40 \%$ de la fuerza laboral y representa el $50 \%$ de las divisas del país. Para 1990 , el área utilizada en cultivos fue de $3 ' 855.000$ hectáreas, de las cuales el $50 \%$ fue ocupada por cultivos tecnificados.

En 1994, la producción nacional de plaguicidas en ingredientes activos superó los 28 millones de kilogramos equivalentes a $53^{\prime} 386.744 \mathrm{~kg} / \mathrm{L}$ de productos comerciales, siendo los fungicidas los de mayor producción $(51,3 \%)$, siguiéndole los herbicidas $(36,0 \%)$, los insecticidas $(11,0 \%)$, los coadyuvantes $(1,6 \%)$, los piretroides $(0,2 \%) \mathrm{y}$, finalmente, los reguladores fisiológicos, con menos de $0,1 \%$. Es importante aclarar que el grupo de los insecticidas incluyen los acaricidas, los molusquicidas, los nematicidas, los desinfectantes del suelo y los fumigantes (6).

En relación con los insecticidas, la producción en ingredientes activos, para 1994, fue de 3'084.750 kg; los OF ocuparon el primer lugar en 
materia de producción al igual que de importación de insecticidas. Con respecto a la comercialización de éstos, se han ido incrementando a partir de 1992 y en un término de 3 años prácticamente se han duplicado (6).

Las ventas en producto comercial para 1993 y 1994 fueron en promedio $35^{\prime} 379.108 \mathrm{~kg}$, ocupando los insecticidas como OF y C, herbicidas y fungicidas, el $97,9 \%$ de la produc-ción y de las ventas de los plaguicidas. Dentro de los países latinoamericanos, Colombia es el tercer país después de Brasil y México en el consumo de plaguicidas (6-8).

En el país, la comercialización de plaguicidas ha adquirido mucha importancia ya que se han venido registrando significativos incrementos en los últimos años, dirigidos a los países del Grupo Andino, Centroamérica y Estados Unidos, principalmente.

Con relación a las estadísticas sobre intoxicaciones tanto agudas como crónicas de OF y $\mathrm{C}$, han sido deficientes debido a la ausencia de un requerimiento unificado a nivel nacional y a la escasa capacitación del personal médico y paramédico (7).

Aún así, se han registrado en el país algunos casos de intoxicación aguda principalmente por OF, como el ocurrido en 1967 en Chiquinquirá, en donde se produjo una contaminación de harina de trigo con paratión, resultando 165 personas intoxicadas, de las cuales murieron 67. En Pasto, en 1976, el consumo de trigo contaminado con paratión, dejó un saldo de 300 intoxicados. En la región de El Espinal, durante los últimos 5 años, se ha producido un promedio de 56 intoxicados por año, de acuerdo a los datos reportados por el hospital local.

Debido al gran uso de estos plaguicidas, el Ministerio de Salud expidió el decreto 1843 del 22 de julio de 1991, referente a disposiciones sanitarias sobre el uso y manejo de plaguicidas y mediante el artículo 170, estableció la notificación obligatoria de todo caso de intoxicación o accidente ocasionado a causa de estos productos (9).

\section{Materiales y métodos}

El Laboratorio de Salud Ambiental y el Servicio de Epidemiología Aplicada del INS analizaron la información recolectada a través del programa de Vigilancia Epidemiológica de Plaguicidas, con el objeto de determinar la prevalencia de intoxicación por plaguicidas OF y $\mathrm{C}$ en 17 departamentos del país de 1993 a 1995. Además, se establecieron los niveles de colinesterasa en la población ocupacionalmente expuesta a plaguicidas e igualmente se determinaron los departamentos, las actividades económicas y los oficios en los cuales se presentó el mayor riesgo de intoxicación.

Se efectuó un estudio descriptivo en el que participaron los trabajadores expuestos ocupacionalmente a los plaguicidas inhibidores de la colinesterasa en los siguientes departamentos del país: Atlántico, Bolívar, Boyacá, Caldas, Cesar, Córdoba, Huila, Magdalena, Meta, Nariño, Norte de Santander, Quindío, Santander, Sucre, Tolima, Valle y la Secretaría de Salud de Bogotá.

La población se obtuvo mediante un censo de empresas dentro de las diferentes actividades económicas y de trabajadores, realizado por los funcionarios de las seccionales de salud de cada uno de los departamentos participantes, a través de las secciones de salud ocupacional, saneamiento ambiental o epidemiología.

Las variables utilizadas en este estudio fueron: identificación general, departamento, regional, municipio y localidad donde labora el trabajador, edad y sexo, actividad económica, oficio y afiliación a la seguridad social, al igual que el análisis de la acetilcolinesterasa, realizado mediante una técnica colorimétrica para la cuantificación de la enzima en sangre total, utilizando el equipo Lovibond el cual es portátil y se puede llevar a cada una de las empresas participantes.

Se establecieron las siguientes categorías:

- Colinesterasa igual o mayor a $75 \%$ de actividad normal.

- Colinesterasa menor de $75 \%$ de actividad. anormal.

La sensibilidad y especificidad del método Lovibond es de $95 \%$ y $100 \%$, respectivamente, valores hallados en el estudio de comparación de cuatro métodos de acetilcolinesterasa que 
consistió en analizar y comparar 190 muestras por los métodos de Merckotest, Michel en plasma, Michel y Aldrige en sangre total y Lovibond, en donde 30 muestras fueron inhibidas con diazinón a diferentes concentraciones.

La fuente de datos estuvo constituida por un formulario el cual se diligenció a cada uno de los trabajadores y que contiene las variables citadas anteriormente.

Los funcionarios de las seccionales de salud se desplazaron a las empresas para la realización de la encuesta y la toma de las muestras de sangre, las que se efectuaron por punción capilar de la yema de los dedos o por punción venosa. Los análisis correspondientes se efectuaron en los laboratorios de las seccionales de salud de cada departamento o en las empresas correspondientes, dependiendo del tiempo de toma de la muestra y de la temperatura ambiente.

Se definió como caso de intoxicación por plaguicidas organofosforados y carbamatos a aquellos trabajadores que presentaron un valor de colinesterasa menor de $75 \%$ de actividad.

Para el análisis de la información, se relacionaron las variables independientes y dependientes, obteniendo las distribuciones de frecuencias agrupadas y se llevó a cabo la estandarización de tasas.

\section{Resultados}

Entre 1993 y 1995, participó una población total de 41.899 personas, las cuales tenían antecedentes de exposición a plaguicidas OF o C. En 1993, se estudiaron 16.299 personas; para 1994, 15.295, y 10.305 para 1995 (figura 1).

En lo que respecta al análisis por edad y género, no se tomó la totalidad de la población ya que, para el primer semestre de 1993, no se habían incluido dentro de la encuesta estas variables. De una población de 33.315 personas, $80 \%$ (26.751) pertenecían al sexo masculino y $20 \%$ al sexo femenino (6.564). Con relación a los grupos de edad, se observó que las mayores prevalencias de valores anormales de ACHE tanto para el sexo masculino como para el femenino se encontraron en el grupo de 18 a 25 años. En general, para ambos sexos, los grupos de mayor riesgo estuvieron entre los 18 y los 40 años.

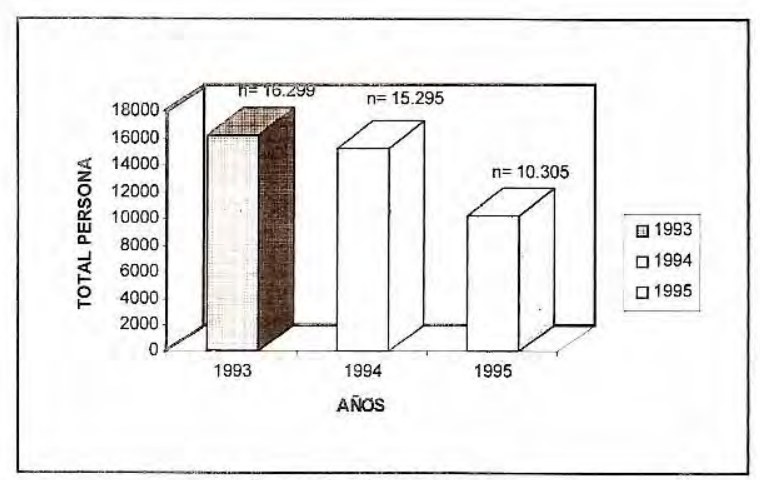

Fuente: Programa de Vigilancıa Epidemiológica, VEO, Instituto Nacional de Salud

Figura 1. Total de personas analizadas expuestas a plaguicidas organofosforados y carbamatos.

Los análisis de las pruebas de colinesterasa frente a la actividad económica mostraron que las mayores prevalencias de valores anormales de ACHE para 1993, fueron para la aplicación urbana con $8,8 \%$, seguido de otras actividades no contempladas en el formato de encuesta, con un $6,1 \%$ y el tercer lugar lo ocupó la aplicación aérea y terrestre con un 5,2 y $5,1 \%$, respectivamente. Para 1994, se halló $18,7 \%$ para las actividades de formulador, seguido de floricultura con $17,7 \%$ y, en tercer lugar, la aplicación aérea con $17,4 \%$. En 1995, las actividades de mayor riesgo estuvieron en la aplicación urbana con $26,1 \%$, seguido de formulador con $16,7 \%$ y el tercer lugar es ocupado por otras actividades con $13,3 \%$. Las actividades económicas que presentaron mayor prevalencia de valores anormales para ACHE, durante el período de 1993 a 1995, correspondieron a la aplicación urbana, formulador y aplicación aérea (cuadro 1).

Con relación a los oficios, durante 1993, las mayores prevalencias se encontraron en el grupo de furnigador-aplicador con $10,7 \%$ y el segundo lugar lo ocupó el grupo de las secretarias que laboran en depósitos o almacenes de agroquímicos con $7,6 \%$. Para 1994, se halló que los grupos más afectados fueron el de piloto-aviador con $21,1 \%$, siendo el segundo lugar para el grupo de mezclador-tanqueador-formulador con $17,3 \%$. En 1995, se presentó en primer lugar el grupo de mezclador-tanqueador-formulador con $25,6 \%$ y 
Cuadro 1. Pruebas de colinesterasa según actividad económica. Colombia, 1993-1995.

\begin{tabular}{|c|c|c|c|c|c|c|c|c|c|c|c|c|}
\hline \multirow{3}{*}{ Actividad ecónomica } & \multicolumn{4}{|c|}{1993} & \multicolumn{4}{|c|}{1994} & \multicolumn{4}{|c|}{1995} \\
\hline & \multirow{2}{*}{\multicolumn{2}{|c|}{$\begin{array}{l}\text { Normal } \\
\mathbf{n}\end{array}$}} & \multirow{2}{*}{\multicolumn{2}{|c|}{$\begin{array}{l}\text { Anormal } \\
\mathrm{n} \%\end{array}$}} & \multicolumn{2}{|c|}{ Normal } & \multicolumn{2}{|c|}{ Anormal } & \multicolumn{2}{|c|}{ Normal } & \multicolumn{2}{|c|}{ Anormal } \\
\hline & & & & & $\mathbf{n}$ & $\%$ & $\mathbf{n}$ & $\%$ & $\mathrm{n}$ & $\%$ & n & $\%$ \\
\hline Formulador & - & - & - & - & 26 & 81,3 & 6 & 18,7 & 5 & 83,3 & 11 & 6,7 \\
\hline Granja experimental & 95 & 100 & - & - & - & - & - & - & 97 & 97,9 & 2 & 2,1 \\
\hline Distribuidor & 2.025 & 97,4 & 53 & 2,6 & 1.590 & 91,9 & 139 & 8 & 1.286 & 95,7 & 58 & 4,3 \\
\hline Aplicación área & 275 & 94,8 & 15 & 5,2 & 214 & 82,6 & 45 & 17,4 & 158 & 87,8 & 22 & 12,2 \\
\hline Aplicación terrestre & 6.932 & 94,9 & 369 & 5,1 & 5.117 & 94,3 & 306 & 5,7 & 1713 & 92,3 & 143 & 7,7 \\
\hline Aplicación urbana & 249 & 91,2 & 24 & 8,8 & 341 & 85,7 & 57 & 14,3 & 133 & 73,9 & 47 & 26,1 \\
\hline Aplicación sanitaria & 92 & 98,9 & 1 & 1,1 & 156 & 95,7 & 7 & 4,3 & 348 & 93,2 & 28 & 6,8 \\
\hline Hacienda finca & 7.031 & 95,4 & 340 & 4,6 & 7.778 & 94,1 & 490 & 5,9 & 5.048 & 93,5 & 355 & 6,5 \\
\hline Floricultura & 83 & 95,4 & 4 & 4,6 & 292 & 82,3 & 63 & 17,7 & 36 & 100 & - & - \\
\hline Otras actividades & 309 & 93,9 & 20 & 6,1 & 240 & 84,9 & 43 & 15,1 & 546 & 86,7 & 84 & 13,3 \\
\hline Total & 17.091 & 95,4 & 826 & 4,6 & 15.754 & 93,2 & 1.156 & 6,8 & 9.442 & 92,7 & 740 & 7,3 \\
\hline
\end{tabular}

Fuente: Programa de vigilancia epidemiológica-VEO- Instituto Nacional de Salud.

el segundo lugar el grupo de profesionales de diferentes ramas con $13,9 \%$. Es importante resaltar que las mayores prevalencias de valores anormales de acetilcolinesterasa para el período 1993 a 1995 se vieron para los oficios de mezcladortanqueador-formulador, profesionales de diferentes ramas, fumigador-aplicador y piloto-aviador (cuadro 2).
El programa cubre 17 departamentos del país y dentro de éstos se distribuye el $57 \%$ en regionales, el $16,2 \%$ en municipios y el $1,1 \%$ en localidades. Los departamentos dentro del programa que mayor continuidad han tenido son: Antioquia, Boyacá, Santander, Norte de Santander, Tolima, Sucre y Valle.

Cuadro 2. Total de pruebas de colinesterasa por oficio. Colombia, 1993-1995.

\begin{tabular}{|c|c|c|c|c|c|c|c|c|c|c|c|c|}
\hline \multirow{3}{*}{ Oficios } & \multicolumn{4}{|c|}{1993} & \multicolumn{4}{|c|}{1994} & \multicolumn{4}{|c|}{1995} \\
\hline & \multicolumn{2}{|c|}{ Normales } & \multicolumn{2}{|c|}{ Anormales } & \multicolumn{2}{|c|}{ Normales } & \multicolumn{2}{|c|}{ Anormales } & \multicolumn{2}{|c|}{ Normales } & \multicolumn{2}{|c|}{ Anormales } \\
\hline & $n$ & $\%$ & $n$ & $\%$ & $\mathbf{n}$ & $\%$ & $\mathbf{n}$ & $\%$ & $\mathbf{n}$ & $\%$ & & $\%$ \\
\hline Cargo administrativo & 9.099 & 94,5 & 531 & 5,5 & 814 & 95,1 & 42 & 4,9 & 829 & 95,9 & 35 & 4,1 \\
\hline Secretaria & 73 & 92,4 & 6 & 7,6 & 263 & 91,0 & 26 & 9,0 & 168 & 92,8 & 13 & 7,2 \\
\hline Almacenista-bodeguero & 47 & 98,7 & 2 & 1,3 & 389 & 91,5 & 36 & 8,5 & 290 & 95,1 & 15 & 4,9 \\
\hline Conductor & 115 & 99,1 & 1 & 0,9 & 126 & 96,2 & 5 & 3,8 & 164 & 95,9 & 7 & 4,1 \\
\hline Vendedor & 65 & 100 & - & - & 247 & 90,1 & 27 & 9,9 & 256 & 97,3 & 7 & 2,7 \\
\hline Agrónomo y prof. afines & 857 & 94,2 & 53 & 5,8 & 204 & 97,1 & 6 & 2,9 & 377 & 95,7 & 17 & 4,3 \\
\hline Prof. diferentes ramas & 26 & 92,9 & 2 & 7,1 & 46 & 88,5 & 6 & 11,5 & 62 & 86,1 & 10 & 13,9 \\
\hline Fumigador-aplicador & 510 & 89,3 & 61 & 10,7 & 3.831 & 91,8 & 341 & 8,2 & 3.511 & 92,7 & 278 & 7,3 \\
\hline Jornalero & 4.428 & 97,4 & 120 & 2,6 & 4.384 & 93,1 & 324 & 6,9 & 3.259 & 92,7 & 2.57 & 7,3 \\
\hline Piloto aviador & 19 & 95,0 & 1 & 5,0 & 15 & 78,9 & 4 & 21,1 & 13 & 100 & - & - \\
\hline Mezclador-tanque.-formu. & 30 & 100 & - & - & 124 & 82,7 & 26 & 17,3 & 67 & 74,4 & 23 & 25,6 \\
\hline Banderero & 12 & 100 & - & - & 1 & 100 & - & - & 1 & 100 & - & - \\
\hline Profesor & 24 & 100 & - & - & 107 & 98,2 & 2 & 1,8 & 19 & 100 & - & - \\
\hline Estud. Esc. Menor & 328 & 97,3 & 9 & 2,7 & 3.279 & 95,5 & 154 & 4,5 & 1.002 & 95,9 & 43 & 4,1 \\
\hline Ama de casa & 507 & 97,1 & 15 & 2,9 & 1.656 & 95,2 & 83 & 4,8 & 598 & 97,0 & 19 & 3,0 \\
\hline Serv. Generales campo & 524 & 99,2 & 4 & 0,8 & 266 & 94,7 & 15 & 5,3 & 128 & 90,8 & 13 & 9,2 \\
\hline Otros & 341 & 98,0 & 7 & 2,0 & 2 & 100 & - & - & 39 & 95,1 & 2 & 4,9 \\
\hline Total & 17.105 & 95,5 & 812 & 4,5 & 15.754 & 93,5 & 1.097 & 6,5 & 10.783 & 93,6 & 739 & 6,4 \\
\hline
\end{tabular}

Fuente: Programa de Vigilancia Epidemiológica, VEO, Instituto Nacional de Salud. 
Se realizó una estandarización directa de las proporciones de anormalidades de colinesterasa por oficio, con el fin de poder llevar a cabo una comparación entre los departamentos participantes en el estudio, lo que permitió encontrar los siguientes datos.

Para 1993, las mayores prevalencias de valores anormales de ACHE por oficio, se encontraron en Huila con $55,9 \%$, seguido de Santander con $48,4 \%$. En 1994, se presentaron en los departamentos de Bolívar con 23,8\% y Meta con $17,3 \%$, y para 1995 , Santander y Córdoba mostraron las mayores prevalencias con $7,3 \%$ y $5,7 \%$, respectivamente.

Se realizó un total de 46.290 pruebas de colinesterasa, encontrándose 5.370 pruebas anormales, equivalentes a $11,6 \%$. Con relación a la población total, se efectuó por persona en promedio 1,1 prueba de colinesterasa al año (figura 2).

En lo que concierne a la seguridad social, sólo se registraron datos de 23.917 personas, de las cuales $68,3 \%$ (16.338) no estaba afiliada a la seguridad social, $24,1 \%$ (5.762) eran cotizantes del ISS y $7,6 \%$ (1.817) pertenecían a otra entidad de seguridad social (figura 3 ).

\section{Discusión}

En Colombia, la agricultura tecnificada se viene practicando desde hace unos 40 años, aunque en los últimos ha tenido su mayor auge por el

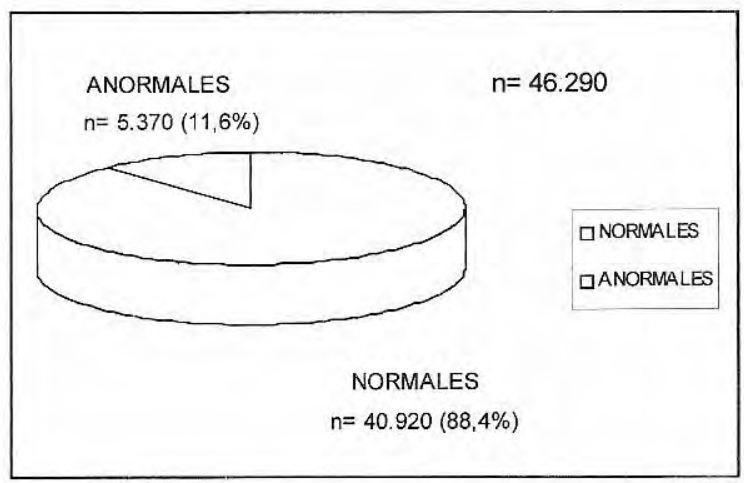

Fuente: Programa de Vigilancia Epidemiológica, VEO, Instituto Nacional de Salud.

Figura 2. Total de pruebas de colinesterasa realizadas durante el período de 1993 a 1995.

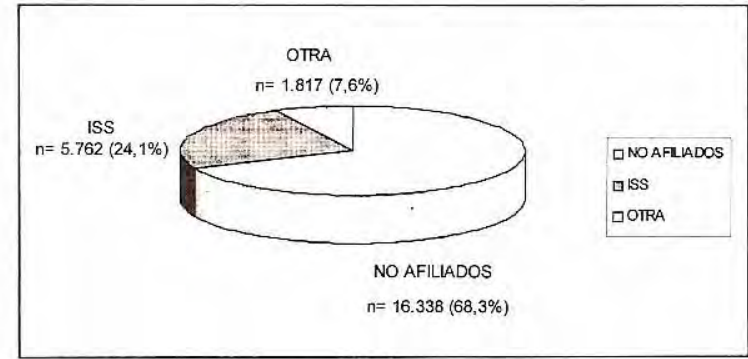

Fuente: Programa de Vigilancia Epidemiológica, VEO, Instituto Nacional de Salud.

Figura 3. Cobertura de seguridad social durante el período de 1993 a 1995.

interés de diversificar las importaciones. El uso intensivo e indiscriminado de insumos agrícolas con el fin de incrementar la producción de los alimentos viene generando innumerables problemas sobre la salud de la población y sobre el medio ambiente, la mayoría de los cuales no han sido evaluados.

Es precisamente en esta actividad en la cual los plaguicidas presentan una eficiente ayuda. Sin embargo, deben ser utilizados en forma adecuada para evitar los riesgos de intoxicaciones que conlleva el uso de estos compuestos químicos.

Actualmente, sólo 17 departamentos se hallan vinculados al Programa de Vigilancia Epidemiológica, lo que impide tener un panorama nacional que nos permita establecer la verdadera magnitud de la intoxicación por OF y lo que es más importante la cantidad de estas sustancias que son utilizadas en los departamentos que no participan y el impacto ambiental que éstas están causando en ellos.

En los datos obtenidos en el estudio, se observó una marcada diferencia en la proporción de intoxicados de acuerdo con el sexo, $80 \%$ correspondió al género masculino; respecto a la edad, las mayores prevalencias de valores anormales se encontraron en el grupo de 18 a 25 años, lo que sugiere que el riesgo de intoxicación por OF y C es más alto para los hombres, presentándose en la etapa de mayor productividad de la vida, en el ambiente ocupacional.

Las actividades dedicadas a la aplicación urbana y aérea y formulador y los oficios de mezclador- 
tanqueador-formulador y fumigador-aplicador, entre otros, fueron los grupos de mayor riesgo ya que presentaron las más altas prevalencias de valores anormales para ACHE.

Vemos cómo los grupos más afectados por estas intoxicaciones son las personas que tienen contacto directo con estas sustancias, hecho éste que pudo ser demostrado por la determinación de colinesterasa, siendo la mayor parte de estas intoxicaciones debidas a la falta de conocimiento para el manejo de estos productos. De la población que se encuentra expuesta a este tipo de tóxicos, $68,3 \%$ no tiene ningún tipo de seguridad social, lo que agrava la situación de estos trabajadores y hace pensar que los diagnósticos por falta de atención oportuna se han hecho tardíamente o, lo que es más grave, no se detecten estos casos y el subregistro puede ser demasiado alto impidiendo conocer la verdadera magnitud del problema.

Desde el advenimiento de los OF y C, éstos se han convertido en las principales sustancias productoras de intoxicaciones agudas por plaguicidas en nuestro país; es por esto que se hace necesario mejorar los programas existentes de salud ocupacional a nivel de las empresas que manejan estas sustancias, como fortalecer la vigilancia epidemiológica y el desarrollo de acciones de promoción, prevención y control que disminuyan la incidencia de este tipo de accidentes a nivel nacional.

Es importante la creación de una conciencia en los directivos de las empresas que manejan estos productos, que vaya orientada a fortalecer sus programas de salud ocupacional, con el objeto de forjar en sus empleados hábitos saludables que los estimulen a manejar todas las normas de seguridad que se han establecido para la manipulación de estas sustancias. Es por esto que el Ministerio de Salud y el Instituto Nacional de Salud a través del programa VEO, se han convertido en el bastión fundamental para el control de este fenómeno y su objetivo primordial es producir cambios en la conducta de las personas directamente involucradas, a través de la educación impartida por los responsables de este programa en cada uno de los departamentos, al igual que detectar precozmente los casos de intoxicación por plaguicidas a través de la determinación de acetilcolinesterasa.

Es indispensable mantener la continuidad de los funcionarios que manejan el programa VEO al interior de los servicios de salud del país ya que los cambios constantes entorpecen el funcionamiento de éste al no tener siempre personal capacitado al frente del mismo. Razones éstas que hacen necesario como primera medida fortalecer el programa a nivel nacional, logrando el ingreso de los departamentos faltantes del pais e, igualmente, intensificando las acciones de prevención y promoción para el manejo de estas sustancias por parte de las secretarías departamentales, las EPS, las IPS y las empresas donde se manipulan plaguicidas, a través de sus programas de salud ocupacional.

Es necesario resaltar que el programa VEO es el único que se lleva a nivel nacional y ha permanecido durante muchos años, aportando datos muy valiosos sobre este tipo de intoxicación, los cuales podrán ser utilizados posteriormente para el desarrollo de futuras investigaciones.

\section{Agradecimientos}

Queremos expresar nuestros más sinceros agradecimientos a todos los funcionarios de los Servicios de Salud que participan en el Programa de Vigilancia Epidemiológica de Plaguicidas VEO, por su entusiasmo, colaboración e interés en el desarrollo del mismo.

Igualmente, a los jefes de los Servicios de Salud quienes con su apoyo, han permitido la capacitación del personal de salud para la continuidad del programa.

\section{Referencias}

1. Cassaret L, Doull J. Toxicology: the basic science of poisons. Fourth edition. New York: Macmillan Publishing Co.; 1991.

2. Dreisbach R. Manual de envenenamientos. Tercera edición. México: Editorial El Manual Moderno; 1978.

3. Fernández G. Intoxicación por plaguicidas. Montevideo: Editorial Monteverde; 1970.

4. Hayes W. Pesticides studied in man. Baltimore/ London: Williams \& Wilkins; 1982. 
5. Centro Panamericano de Ecología Humana y Salud, OPS/OMS. Prevención, diagnóstico y tratamiento de intoxicaciones por insecticidas; 1986.

6. Instituto Colombiano Agropecuario. Comercialización de plaguicidas 1994-1995. ImportaciónProducción-Ventas-Exportación. Santa Fe de Bogotá: ICA; 1997.

7. Ministerio de Salud. Los plaguicidas en América Latina. Salud Ambiente y desarrollo. Segunda edición. Santa Fe de Bogotá: Ministerio de Salud; 1994;21527.

8. Morales C. Los plaguicidas agrícolas en Colombia. Ministerio de Agricultura, Instituto Colombiano Agropecuario, 1992.

9. Ministerio de Salud. Disposiciones sanitarias sobre uso y manejo de plaguicidas. Decreto $1843 \mathrm{del} 22 \mathrm{de}$ julio de 1991.

\section{Lecturas recomendadas}

1. Ames RG, et al. Chronic neurologic sequelae to cholinesterase inhibition among agricultural pesticide applicators. Arch Environ Health 1995;50:440-4.

2. Banerjee $\mathrm{J}$, et al. Inhibition of human fetal brain acetylcholinesterase: marker effect of neurotoxicity. J Toxicol Environ Health 1991;33:283-90.

3. Burger $\mathbf{M}$, et al. Plaguicidas en medio ambiente. Montevideo: Departamento de publicaciones, Universidad de la República; 1991.

4. Ciesielski S, et al. Pesticide exposures, cholinesterase depression, and symptoms among North Carolina migrant farmworkers. Am J Public Health 1994; 84:446-51.

5. Daniell W, et al. Neuropsychological performance among agricultural pesticide applicators. Environ Res 1992;59:217-28.

6. Forget G. Pesticides and third world. J Toxicol Environ Health 1991;32:11-31.

7. Goswamy R, Chaudhuri A, Mashashur A. Study of respiratory failure in organophosphate and carbamate poisoning. Heart Lung 1994;23:466-72.

8. Herrera GC, et al. Condiciones de higiene, seguridad y efectos en la salud de los trabajadores de las empresas de fumigación domiciliaria que utilizan organofosforados y carbamatos categoría l.

9. Jaramillo ML, Bedoya ME. Condiciones laborales y efectos en la salud por exposición a organofosforados y carbamatos en el cultivo de flores Agrícola Papagayo. Universidad Nacional de Colombia $1985 ; \mathrm{I}-\mathrm{XI} ; 104$.tab.
10. Karr C, et al. Organophosphate pesticide exposure in a group of Washington State orchard applicators. Environ Res 1992;59:229-37.

11. Kishi M, et al. Relationship of pesticide spraying to signs and symptoms in Indonesian farmers. Scand $\mathrm{J}$ Work Environ Health 1995;21:124-33.

12. Levine R, Doull J. Global estimates of acute pesticide morbidity and mortality. Rev Environ Contam Toxicol 1992;129:29-50.

13. Limperos G, Ranta K. The rapid field determination of cholinesterase. Science 1953:117.

14. Lotti M. The pathogenesis of organophosphate polyneuropathy. Crit Rev Toxicol 1991;21:465-87.

15. Matchaba RB, Siziya S. Organophosphate exposure in pesticide formulation and packaging factories in Harare, Zimbabwe. Cent Afr J Med 1995;41:40-4.

16. Matos EL, et al. Efectos de los plaguicidas en trabajadores de cultivos intensivos. Bol Oficina Sanit Panam 1988;104:160-70.

17. McConnell R, Hruska AJ. An epidemic of pesticide poisoning in Nicaragua: implications for prevention in developing countries. Am J Public Health 1993;83:1559-62.

18. Nhachi C, Kasilo O. The pattern of poisoning in urban Zimbabwe. J Appl Toxicol 1992;59:229-37.

19. Roselli $\mathbf{Q}$, et al. Leucoencefalopatía aguda difusa causada por exposición a organofosforados. Acta Med Colomb 1979;4:13-21.

20. Rotenberg $\mathbf{M}$, et al. Differentiation between organophosphate and carbamate poisoning. Clin Chim Acta 1995;234:11-21.

21. Sherman J. Organophosphate pesticidesneurological and respiratory toxicity. Toxicol Industrial Health 1995; 11:33-9.

22. Sidell FR. Clinical effects of organophosphorus cholinesterase inhibitors. J Appl Toxicol 1994;14:1113 .

23. Steenland K, et al. Chronic neurological sequelae to organophosphate pesticide poisoning. Am J Public Health 1994;84:731-6.

24. Sultatos L. Mammalian toxicology of organophosphorus pesticides. J Toxicol Environ Health 1994; 43:271-89.

25. Wesseling C, Castillo L, Elinder CG. Pesticide poisonings in Costa Rica. Scand J Work Environ Health 1993;19:227-35. 